17th International Conference on Computing in High Energy and Nuclear Physics (CHEP09) IOP Publishing Journal of Physics: Conference Series 219 (2010) 032062 doi:10.1088/1742-6596/219/3/032062

\title{
MDT data quality assessment at the Calibration Centres for the ATLAS experiment at LHC
}

\author{
Monica Verducci \\ University of Wuerzburg \\ Am Hubland, 97074, Wuerzburg, Germany \\ E-mail: monica.verducci@cern.ch \\ Elena Solfaroli Camillocci \\ INFN and Università degli Studi di Roma 'La Sapienza' \\ P.le Aldo Moro 500185 Rome, Italy \\ E-mail: elena.solfaroli@roma1.infn.it \\ Valerio Consorti \\ INFN and Università degli Studi di Roma 'La Sapienza' \\ P.le Aldo Moro 500185 Rome, Italy \\ E-mail: valerio.consorti@roma1.infn.it
}

\begin{abstract}
ATLAS is a large multipurpose detector, presently in the final phase of construction at the CERN Large Hadron Collider accelerator. In ATLAS the muon detection is performed by a huge magnetic spectrometer, built with the Monitored Drift Tube technology. It consists of more than 1,000 chambers and 350,000 drift tubes, which have to be controlled to a spatial accuracy better than $10 \mu \mathrm{m}$ and an efficiency close to $100 \%$. Therefore, the automated monitoring of the detector is an essential aspect of the operation of the spectrometer. The quality procedure collects data from online and offline sources and from the Calibration Stream at the Calibration Centres, situated in Ann Arbor (Michigan), MPI (Munich) and INFN Rome. The assessment at the Calibration Centres is performed using the DQHistogramAnalyzer utility of the ATHENA package. This application checks the histograms in an automated way and, after a further inspection with a human interface, reports results and summaries. The analysis results are stored in an Oracle Database using the COOL LCG library, through a C++ object-oriented interface. In this study a complete description of the entire chain, from the calibration stream up to the database storage is presented.
\end{abstract}


17th International Conference on Computing in High Energy and Nuclear Physics (CHEP09) IOP Publishing Journal of Physics: Conference Series 219 (2010) $032062 \quad$ doi:10.1088/1742-6596/219/3/032062

\section{Introduction}

High pressure Monitored Drift Tube (MDT) [1] chambers will be used as precision tracking detectors in the Muon Spectrometer [2] of the ATLAS experiment at the Large Hadron Collider (LHC) at CERN. An accurate knowledge of the space-time relation is needed to reach the design average resolution of about $80 \mu \mathrm{m}$.

The model for the calibration [3] of the MDT chambers defines the optimal number of muon tracks, the procedures and the requirements of the calibration software. This model imposes a substantial effort in data collection, software implementation and data management.

The standard Atlas data-flow cannot provide the required statistics and calibration frequency, therefore an ad hoc mechanism, allowing the extraction of a dedicated data stream [4] for muons (the Muon Calibration Stream), was introduced. The level-2 trigger turned out to be the ideal place to extract muon data.

Data are streamlined and processed in three remote Tier-2 Calibration Centres, situated in Ann Arbor (Michigan), MPI (Munich) and INFN Rome. The computation model foresees that the data are sent to the Calibration Centres synchronously, as soon as they are available from the Calibration Stream. The calibration model described above has been defined and approved by the ATLAS Management and fully tested during the cosmic tests.

The local computation and the data quality checking start almost immediately after the beginning of the data taking. Each Calibration Centre runs the same software and performs a fraction of the total computation, with small overlaps for testing and checking purposes. At the end of the computation, the results (i.e. the assessment of the quality of the data and the calibration constants) are sent back to the central computing facilities at CERN, checked for overlaps, merged and inserted in the ATLAS Conditions database.

\section{The MDT data quality assessment at the Calibration Centre}

The MDT data quality (DQ) assessment is performed at the Calibration Centres using the ATLAS software package DQHistogramAnalyzer. It is also referred to as 'offline DQMF', as the primary application uses the ATLAS Data Quality Monitoring Framework (DQMF) [5], which is a distributed software system providing data quality monitoring functionality in the online environment.

The main purpose of the DQHistogramAnalyzer is to check histograms in an automated way, report results and summaries, and generate alarms when deviations from the standard are encountered.

The detector expert configures the DQMF application, defining the DQ check algorithm for each monitoring histogram. In some cases, a comparison with a reference histogram is requested to perform shape or bin to bin consistency checks.

At the end of the analysis, each histogram is associated with a quality flag (Green, Yellow or Red), according to the check results.

In order to apply a specific analysis for the MDT chambers, some DQ algorithms were developed and added to the generic ones, already available in the package.

In particular, the analysis of the MDT chambers checks the stability of the essential parameters, such as the maximum drift time, the chamber occupancy and the tube efficiency. Then several overview histograms are analysed to check the global detector status. In figure 1 the time spectrum and the tube 
17th International Conference on Computing in High Energy and Nuclear Physics (CHEP09) IOP Publishing Journal of Physics: Conference Series 219 (2010) $032062 \quad$ doi:10.1088/1742-6596/219/3/032062

efficiency for a MDT chamber analysed at the calibration centres are shown.

The MDT DQ results are finally published in a web display, which can be consulted by the shift crew. The main page of the web display shows the chamber identifiers with their final quality flags; clicking on them, the DQ histograms for each chamber and their associated flags become visible. Then clicking on the histogram itself, a brief summary of the results is shown.

Finally the quality flags and the useful parameters (i.e. the maximum drift time) are archived inside a dedicated database for future retrieval.
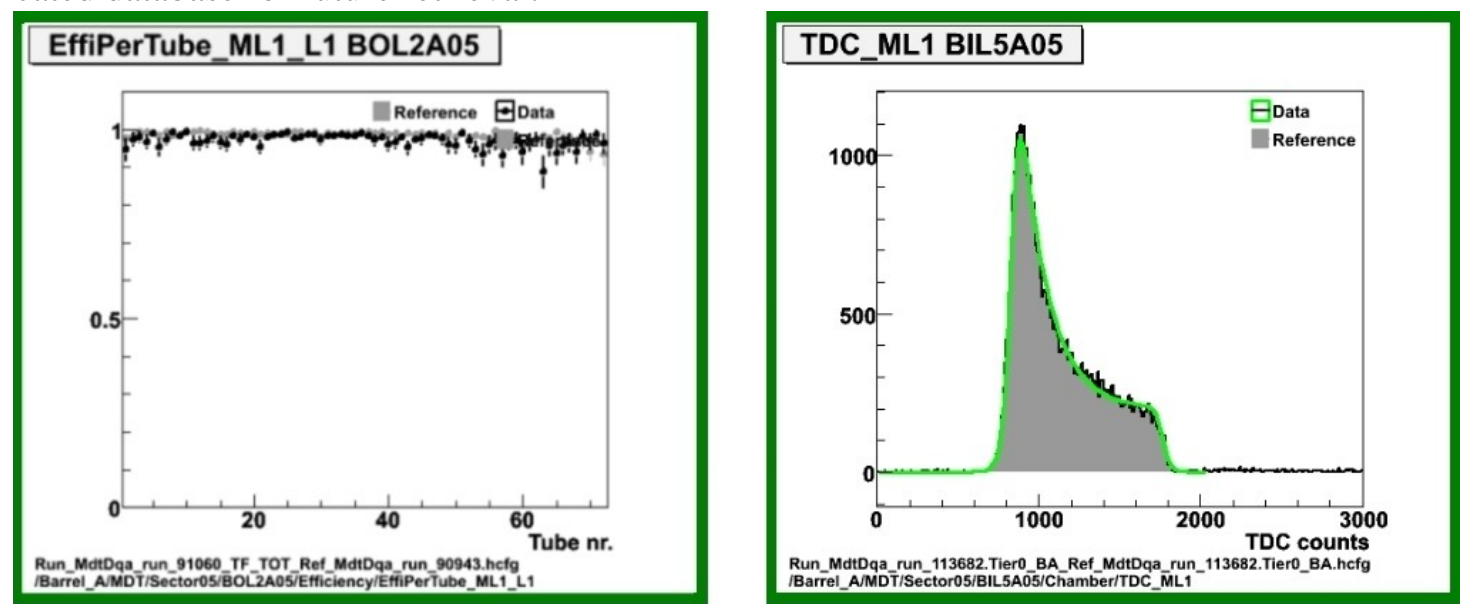

Figure 1. MDT tube efficiency (left) and drift time spectrum (right) after the DQ analysis at the MDT calibration centres. The frame color is the resulted quality flag. The time spectrum is fitted with the function described in [8].

\subsection{The MDT data quality database and dataflow}

The MDT DQ results are stored in a dedicated database schema running on the Tier- 0 at CERN. These conditions parameters are recorded during the ATLAS data taking and used by the reconstruction and analysis. The MDT DQ database schema foresees different tables dedicated to each piece of relevant information. Each table has its own structure, mainly divided into several columns. Each column contains a single parameter (e.g. drift time for multilyer), or an ordered sequence of parameters (e.g. all the dead tubes in a given chamber).

All the MDT chamber information for a particular time interval resides in one row, identified by the chamber 'offline identifier', i.e. the label of the chamber used by the ATHENA reconstruction programs.

The DQMF framework writes directly the 'algorithm results' in the database. It uses Python scripts running after the analysis of the histograms.

The MDT DQ database represents a part of the general MDT dataquality schema placed inside the ATLAS Online Rac (ATONR) server running on Tier-0. For development reasons, we have a test replica inside the Integration Rac (INTR) server running on Tier-0 as well.

The MDT DQ database belongs to the General Conditions Database, in agreement with the ATLAS Database Model [6]. The conditions database is a general database dedicated to the managing of the parameters describing run conditions and logging, i.e. the data which will be accessed offline by the reconstruction or analysis software. These parameters include data archived by the ATLAS detector control system (DCS), online book-keeping data, online and offline calibration and alignment data, and monitoring data characterising the performance of the detector and software during any particular 
17th International Conference on Computing in High Energy and Nuclear Physics (CHEP09) IOP Publishing Journal of Physics: Conference Series 219 (2010) $032062 \quad$ doi:10.1088/1742-6596/219/3/032062

period of time.

\section{The ATLAS Condition Database Design}

The ATLAS Condition Database is based on Oracle DB. It is implemented using the Conditions Objects for LHC (COOL) technology. COOL is an LCG product [7], that allows database applications to be written independently of the underlying database technology (this means that COOL databases can be stored in Oracle, SQLite or MySQL [7]).

Moreover, the COOL API has been integrated into the ATLAS online software. Several specialpurpose higher level interfaces are also being developed, including the Conditions Database Interface (CDI) for archiving Information System (IS) data to COOL, the Process Visualization and control System Software (PVSS) to COOL interface for archiving DCS data, and specialised interfaces for saving monitoring data.

The objects stored or referenced in COOL have an associated start and end time validity. The data are stored via an Interval of Validity (IoV).

The COOL data are stored in folders, which are themselves arranged in a hierarchical structure of folder sets. Within each folder, several objects of the same type are stored, each with its own interval of validity range. These times are specified either as run/event, or as absolute timestamps, and the choice between formats is made according to meta-data associated with each folder. The objects in COOL folders can be optionally identified by a channel number (or channel ID) within the folder. Each channel has its own interval of validity, but all channels can be dealt with bulk updates or retrieves. The retrieving and storing of the data inside a reconstruction job in the Athena framework is possible using the IOVService, an interface between the COOL DB and the reconstruction algorithms via IOV range.

Every Conditions data is stored in data objects which mantain the information about the time validity. For each event the IOV Service checks the interval of these object containers with respect to the current event. In case of expiration of validity it deletes the information in the containers and reloads the last updated data from the COOL DB.

The operation of the ATLAS muon spectrometer requires that the more than 1,000 MDT chambers have to be constantly controlled. Therefore, an automated detector monitor is an essential aspect of this challenge.

\section{Conclusions}

In this paper the procedure for the data quality assessment at the MDT Calibration Centres has been presented. Some MDT specific DQ algorithms were developed and are available in the ATLAS software repository.

At the moment the offline DQMF utility for the MDT DQ assessment is almost completed and already used by the experiment. The DQ results are published daily on the web display and stored in the MDT DQA COOL database.

\section{References}


17th International Conference on Computing in High Energy and Nuclear Physics (CHEP09) IOP Publishing Journal of Physics: Conference Series 219 (2010) $032062 \quad$ doi:10.1088/1742-6596/219/3/032062

[1] Livan M 1996 Monitored drift tubes in ATLAS Nucl. Inst. And Meth. in Phys. Res A384 214

[2] ATLAS Muon Collaboration 1997 ATLAS Muon Spectrometer Technical Design Report CERN/LHCC/97-022

[3] D.Orestano et al. 2008 Calibration model for the MDT chambers of the ATLAS Muon Spectrometer ATL-MUON-PUB-2008-004

[4] E.Pasqualucci et al. 2006 Muon detector calibration in the ATLAS experiment: data extraction and distribution, Proceedings of Computing In High Energy and Nuclear Physics CHEP 2006

[5] A.Corso-Radu et al. 2007 Data Quality Monitoring Framework for the ATLAS Experiment at the LHC, 15th IEEE Real Time Conference 2007

[6] ATLAS Collaboration 2005 ATLAS Computing Technical Design Report CERN/LHCC/2005-022

[7] A. Valassi COOL web page, Available at http://lcgapp.cern.ch/project/CondDB/

[8] P.Creti et al. ATL-MUON-196 (29 June 1997) 